\title{
Zum semiotischen Wert der TV-Ticker
}

\author{
Anna Kapuścińska
}

Online publiziert: 30. März 2016

(C) The Author(s) 2016. This article is available at SpringerLink with Open Access

Zusammenfassung Die Entwicklung der Medien konfrontiert die gegenwärtige Semiotik mit der besonders schwierigen Aufgabe, ihr Interessengebiet auf Gegenstände zu erweitern, die sie bisher nicht untersucht hat. Die Tatsache, dass diese Einheiten den gängigen Zeichen nur teilweise entsprechen, ist sicherlich kein Grund, sie aus der Zeichenkategorie auszuschließen. Die Reflexion über ihren Zeichencharakter und ihr Verhältnis zu anderen Zeichen erfordert jedoch eine vorurteilsfreie Einstellung und einen Zeichenbegriff, der breit genug ist, um neue zeichenhafte Einheiten einzuschließen. Dies betrifft z. B. Nachrichtenticker im Fernsehen, die in diesem Artikel diskutiert werden.

Schlüsselwörter Semiotik · Zeichen · Fernsehen · Nachrichtensendung · TV-Ticker · Geschwindigkeit · Gleichzeitigkeit · Information · Textualität · Bildschirmfläche

\section{On the semiotic value of the news tickers}

Abstract The development of the media confronts contemporary semiotics with the particularly difficult task to broaden its field of interest to include phenomena which have never been investigated before. The fact that such units only partially resemble the current signs is undoubtedly no reason for excluding them from the sign category. However, the reflection on their sign character and their position among other signs requires a prejudice-free approach and a notion of sign which

Zusätzliche Informationen sind in der Online-Version dieses Artikels enthalten: http://dx.doi.org/10. 1007/s41244-016-0007-5.

Dr. A. Kapuścińska $(\square)$

Uniwersytet Kazimierza Wielkiego

Bydgoszcz, Polen

E-Mail: a.kapuscinska@ukw.edu.pl 
is broad enough for the new units. This concerns for example news tickers on television, which are discussed within the framework of this article.

Keywords Semiotics $\cdot$ Sign $\cdot$ Television $\cdot$ TV-news $\cdot$ TV-ticker $\cdot$ Speed · Simultaneousness $\cdot$ Information $\cdot$ Textuality $\cdot$ Screen surface

Als der Entstehungszeitpunkt der Ticker im Fernsehen gilt die ,Revolution“, die im Sommer 2001 bei CNN stattfand. Zu dieser Zeit wurde ein neues Muster der Nachrichtensequenzierung auf dem Bildschirm eingesetzt, in dem das bisherige Bild nach oben verschoben wurde, um unten für den Ticker, d.h. eine „kurze, aktuelle und kontinuierliche Übertragung von Content an ein Empfängergerät“" (Otto/ Scheurer/Vogel 2012, S. 229), Platz zu machen (vgl. Trojanowska/Francuz 2007, S. 67 f.). ${ }^{1}$ Diese Änderung bedeutete noch einen Schritt weiter in Richtung der Nachrichtenbeschleunigung. ${ }^{2}$ Danach wurde dieses Muster von mehreren europäischen Sendern adaptiert. Die Ticker treten inzwischen überwiegend in Spartenprogrammen (vgl. Abb. 1) mit Schwerpunkt Information, aber auch in Vollprogrammen ${ }^{3}$ - unter anderen in Nachrichtensendungen ${ }^{4}$ - (vgl. Abb. 2) auf (vgl. Trojanowska/ Francuz 2007, S. 68).

Auf die TV-Ticker lässt sich die Behauptung von Paul Virilio beziehen, dass die Information heutzutage von ihrer energetischen Beschleunigung untrennbar ist, was Virilio mit einer Szene aus dem politischen Leben Bill Clintons illustriert: Während der Präsidentenwahlen in den Vereinigten Staaten sollte er von seinen Beratern dazu überredet werden, ,so schnell wie möglich zu reden“, um ,,alles Notwendige über ein Thema in weniger als neunzig Sekunden“"zu sagen und es nach seinem Wahlsieg nicht wieder aufgreifen zu müssen (Virilio 2000, S. 70). Dementsprechend ist es das Ziel der TV-Ticker, dass möglichst viel möglichst schnell ,gesagt“" wird, denn sie scheinen dem Prinzip ,schneller, kleiner, günstiger“ (Virilio 2000, S. 63) zumindest auf zweierlei Art und Weise - durch die Geschwindigkeit und Gleichzeitigkeit - zu dienen.

\footnotetext{
1 Ursprünglich wurde die Bezeichnung Ticker in Bezug auf Fernschreiber verwendet, mit denen Nachrichtenagenturen die Nachrichtenmeldungen übermittelten. Dabei entstand beim Empfangsgerät ein tickendes Geräusch, das den Namen des Tickers motiviert hat (vgl. Otto/Scheurer/Vogel 2012, S. 229).

2 Auch früher spielte die Geschwindigkeit bei CNN eine unübersehbare Rolle. Bereits zur Zeit seiner Entstehung stellte ein Journalist die eher rhetorische Frage: „Slow news, no news?“ (vgl. Virilio 2000, S. 127).

3 Hierfür wird die in der Medienwissenschaft gängige Distinktion übernommen. Dabei definiert man das Vollprogramm (sowohl im Funk als auch im Fernsehen) in Anlehnung an den Rundfunkstaatsvertrag vom 31. August 1991 als ein Programm ,mit vielfältigen Inhalten, in welchem Information, Bildung, Beratung und Unterhaltung einen wesentlichen Teil des Gesamtprogramms bilden“ (Staatsvertrag 2012, S. 6; vgl. dazu Marchal 2002, S. 2119) und das Spartenprogramm als ein Programm ,mit im Wesentlichen gleichartigen Inhalten“ (Staatsvertrag 2012, S. 6; vgl. Marchal 2002, S. 2119; vgl. dazu Schwarzkopf 2002, S. 2267).

4 Außer den Nachrichtensendungen werden Ticker auch in Live-Übertragungen, beispielsweise von Sportveranstaltungen, angezeigt (vgl. Otto/Scheurer/Vogel 2012, S. 229). Wegen wesentlicher Unterschiede im situativen Kontext sollte der semiotische Wert solcher TV-Ticker separat untersucht werden. Das ist aber nicht das Thema des vorliegenden Beitrags.
} 


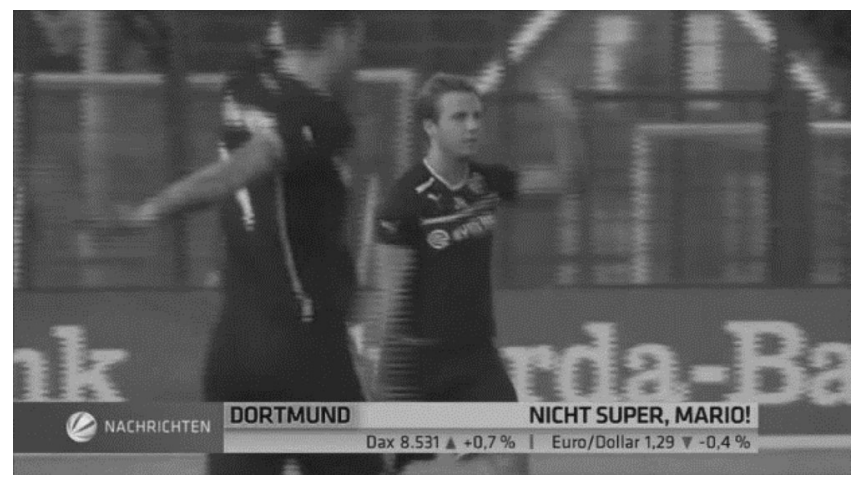

Abb. 1 TV-Ticker in Sat.1 Nachrichten (22.05.2013)

Zweifelsohne ermöglicht die Geschwindigkeit eine wesentliche Informationskompression, was jedoch nicht unbedingt ihrer Rezeptionsweise entspricht. Es wurde zwar nachgewiesen, dass die Zuschauer auf die TV-Ticker unterschiedlich reagieren und dass manche versuchen, ihren Inhalt zu lesen (vgl. Trojanowska/Francuz 2007, S. 68). ${ }^{5}$ Das ist jedoch auf den Aspekt zurückzuführen, den Klaus Brinker (in Bezug auf Texte) als Wirkung bezeichnet, die im Unterschied zur Funktion nicht konventionalisiert ist (vgl. Brinker 2010, S. 89). Offensichtlich sind die TV-Ticker nicht zur textüblichen Rezeption konzipiert, weil eine solche Rezeption durch ihre Form zum großen Teil erschwert wird (vgl. Kapuścińska 2012, S. 115).

Erhebliche Geschwindigkeit beeinträchtigt die Aufmerksamkeit des Empfängers, während die Pluralität gleichzeitig emittierter Informationen Langeweile und Gleichgültigkeit bewirkt (vgl. Cieszkowski 2009, S. 10). Infolgedessen endet die Digitalisierung eher in Desinformation (vgl. Virilio 2000, S. 103). Jean Baudrillard betont in diesem Zusammenhang die umgekehrte Proportionalität zwischen dem Informations- und Sinngehalt. Der Information schreibt er einen zerstörerischen Charakter zu, indem sie den Sinn und die Bedeutung vernichtet. Dabei nennt er zwei Ursachen für diesen Zustand: Erstens erschöpft sich die Information im Inszenieren von Kommunikation, statt tatsächliche Kommunikation zu begünstigen. Zweitens bewirken die Massenmedien die Destrukturierung des sozialen Bereichs, indem die Information den Sinn verliert (vgl. Baudrillard 2006, S. 79-81). ${ }^{6}$

Die Bedeutung des TV-Tickers ist daher eher auf eine binäre Relation der Anwesenheit und Abwesenheit reduziert. Das scheint jede Text-Funktion des Tickers auszuschließen, für die die semantische Bedeutung eine Grundvoraussetzung ist (vgl. Kapuścińska 2012, S. 112). Seine tatsächliche Funktion ist demnach außerhalb

\footnotetext{
5 Abgesehen von den individuellen Unterschieden in der Reaktion auf die TV-Ticker lässt sich vermuten, dass die Zuschauer, die die Sendung nicht von Anfang an gesehen haben, mit größerer Wahrscheinlichkeit die Einträge lesen, um sich darüber zu informieren, was vorher gesagt wurde (vgl. Kapuścińska 2012, S. 115).

6 Einen weiteren Grund für Desinformation nennt Neil Postman, der sie mit dem Unterhaltungscharakter der Fernsehnachrichten verbindet: „Desinformation bedeutet irreführende Information - unangebrachte, irrelevante, bruchstückhafte oder oberflächliche Information -, Information, die vortäuscht, man wisse etwas, während sie einen in Wirklichkeit vom Wissen weglockt“" (Postman 2008, S. 133).
} 


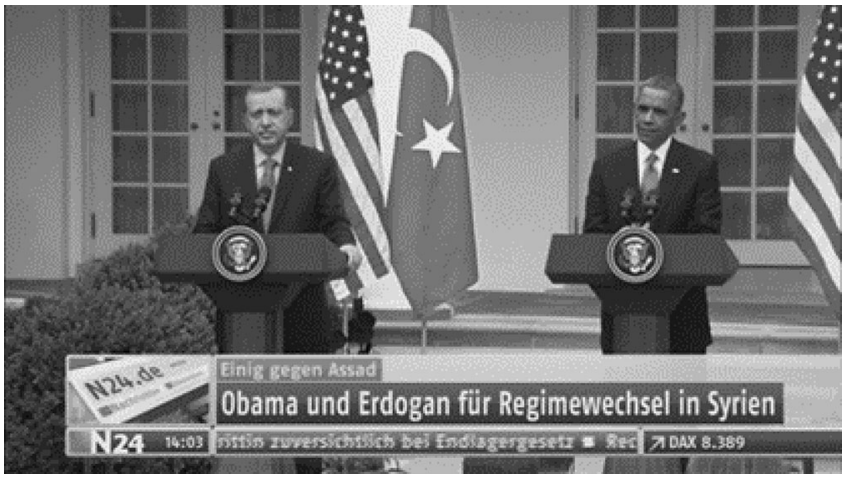

Abb. 2 TV-Ticker in N24 Nachrichten (17.05.2013)

der klassischen Typologien von Textfunktionen zu suchen. Die meistens genannten Funktionen des TV-Tickers lassen sich zumindest in zwei Bereiche unterteilen: in die empfängerbezogenen und die umfeldbezogenen Funktionen.

$\mathrm{Zu}$ den Funktionen, die empfängerbezogen sind, zählt die von Trojanowska und Francuz genannte Funktion, dem Empfänger das Gefühl zu geben, dass er laufend informiert wird. Dabei entspricht dieses Gefühl nicht unbedingt der Wirklichkeit, weil sich der Empfänger nur eine begrenzte Menge der Informationen merken kann (vgl. Trojanowska/Francuz 2007, S. 67 f.). Cieszkowski spricht dabei vom „Suggerieren“ oder sogar „Simulieren“ der Realität. Derartige Einheiten (als ,,virtuelle Text-Bilder" bezeichnet) verfügen nämlich immer häufiger über keinen Referenten, und daher besteht ihre repräsentative Funktion im vorläufigen Dasein, Manifestieren zeitweiliger Anwesenheit (Cieszkowski 2009, S. 10). Dadurch wird der TVTicker zum Simulakrum des Informiertseins. Angesichts der Desinformation tritt das Informiertsein in den Bereich der Hyperrealität. Baudrillard zufolge

geht [es] nicht mehr um Imitation, um die Verdoppelung oder um die Parodie.

Es geht um die Substituierung des Realen durch Zeichen des Realen, d.h.

um eine dissuative Operation, um die Dissuasion realer Prozesse durch ihre operative Verdoppelung, eine programmatische, fehlerlose Signalmaschinerie, die sämtliche Zeichen des Realen und Peripetien (durch Kurzschließen) erzeugt (vgl. Baudrillard 1978, S. 9).

Gleichzeitig wird noch eine Funktion genannt, die in den komplexen Interaktionen mit anderen virtuellen Text-Bildern besteht (vgl. Cieszkowski 2009, S. 2) und daher eher als umfeldbezogen einzustufen ist. In diesem Zusammenhang wird an die Konzeption von Roy Harris angeknüpft, der drei Arten von semiologischen Relationen zwischen dem Text und der Fläche unterscheidet:

- der Text determiniert die Auswahl der Fläche, indem die Bedeutung des Textes von der Fläche abhängt;

- der Text setzt bestimmte physische Merkmale der Fläche voraus, wobei die beiden Komponenten in funktionaler Beziehung zueinander stehen; 
- der Text ist ein Kommentar zur Fläche, auf der er sich befindet (vgl. Harris 1995, S. 114; nach Cieszkowski 2009, S. 5).

Ebenso steht der TV-Ticker in komplexen Relationen zur Fläche. Während der Inhalt der Einträge jeweils einmalig ist und den aktuellen Ereignissen entspricht, ist die Lokalisierung des TV-Tickers auf der Fläche sowie seine Geschwindigkeit ein konstanter Parameter. Diese Tatsache trägt zur Homogenisierung der Nachrichten bei. Dell Champlin und Janet Knoedler zufolge führt eine Analyse der Nachrichten nach dem 11. September 2001 zur Schlussfolgerung, dass nahezu alle Veränderungen in den Nachrichtensendungen ausschließlich den Krieg betreffen, was den Eindruck ergibt, dass sich nur das Thema der Nachrichten verändert hat, während die Einstellung zu ihnen gleich bleibt (vgl. Champlin/Knoedler 2002, S. 464).

Aus der Betrachtung von Funktionen, die den TV-Ticker zugeschrieben werden, ergibt sich, dass sie keineswegs mit Texten gleichzusetzen sind. ${ }^{7}$ Vielmehr soll man sie als Bilder betrachten, und zwar nicht als irgendwelche Bilder, sondern als ideale Bilder, die sich auf die mathematische Vollkommenheit des Systems stützen und dessen Hegemonie unterliegen (vgl. Cieszkowski 2009, S. 7 f.). Die in kulturkritischer Perspektive verwurzelte Feststellung, dass der TV-Ticker kein textuelles und daher auch kein sprachliches Zeichen ist, hält den Forscher von den ausgetretenen wissenschaftlichen Wegen der Text-Zeichenhaftigkeit fern und lässt ihn einen kaum benutzten Weg wählen.

Bei der Reflexion über den semiotischen Wert des TV-Tickers soll davon ausgegangen werden, was man unter Zeichen versteht. Zweifelsohne ist das ein polysemer Terminus, dessen Definition je nach Forschungsparadigma variieren kann. Die Basis für die nachfolgenden Überlegungen ist die Zeichendefinition von Umberto Eco, einem der namhaftesten Vertreter der Peirce'schen Strömung in der Semiotik. Eco definiert Zeichen wie folgt:

Ein Zeichen liegt dann vor, wenn durch Vereinbarung irgendein Signal von einem Kode als Signifikant eines Signifikats festgelegt wird. Ein Kommunikationsprozeß liegt vor, wenn ein Sender bewußt kodierte Signale mittels eines Sendegeräts überträgt, das sie über einen Kanal schickt; die Signale aus dem Kanal werden von einem Empfangsgerät empfangen, das sie in eine Botschaft umwandelt, die ein Empfänger erfassen kann, der dann aufgrund des Kodes mit der Botschaft als der signifikanten Form ein Signifikat oder einen Inhalt der Botschaft verbindet. Wenn der Sender nicht intentional sendet und als natürliche Quelle erscheint, handelt es sich um einen Designationsprozeß vorausgesetzt die übrigen Elemente sind gegeben.

Ein Zeichen ist eine Korrelation eines Signifikanten mit einer Einheit (oder einer Hierarchie von Einheiten), die wir als Signifikat definieren. In diesem Sinn ist das Zeichen immer semiotisch autonom gegenüber den Gegenständen, auf die es bezogen werden kann (Eco 1977, S. 167).

\footnotetext{
${ }^{7}$ Der Text-Begriff wird dabei nach Cieszkowski auf das Sprachliche beschränkt (vgl. u. a. Cieszkowski 2009, S. 11) und als „,begrenzte Menge miteinander verknüpfter Sprachzeichen“ (Cieszkowski 2001, S. 113) konzeptualisiert.
} 
Der mehrmals formulierte Einwand, dass es demnach ,prinzipiell nichts auf der Welt (gibt), was nicht für Rezipienten ,Bedeutung ' haben und somit ,Zeichen“ sein könnte“ (Trabant 1996, S. 83), ist vor allem in Bezug auf die TV-Ticker eher als ein Vorteil zu betrachten, weil sie dadurch nicht allzu vorläufig aufgrund mangelnder Textualität oder ihrer vermeintlich intentionslosen automatisierten Produktion aus der Zeichen-Kategorie ausgegrenzt werden.

Im Folgenden wird die Kategorie der TV-Ticker durch das Prisma dieser Definition betrachtet, indem sowohl der Frage nachgegangen wird, ob sie Zeichen sind, als auch - bei einer positiven Antwort auf die erste Frage -, ob sie als Zeichen einen kommunikativen Wert haben.

Die vorliegende Diskussion bezieht sich auf den gesamten TV-Ticker, der aus (1) dem Streifen auf der Fläche des Bildschirms, (2) dem sprachlichen Text und (3) der Geschwindigkeit besteht. Alle drei Elemente werden als Figuren betrachtet. Der Begriff Figur ist auf die Konzeption der Gliederung des ikonischen Codes von Luis Prieto zurückzuführen. Dabei konstituiert die ,erste Gliederung“ die Ebene der Zeichen, als der die Elemente angesiedelt sind, die Bedeutung denotieren, während sich die ,zweite Gliederung“ auf Figuren bezieht, ,die keine Faktoren der (...) denotierten Bedeutung darstellen“ (vgl. Eco 2002, S. 236) ${ }^{8}$. Eco zufolge sind Figuren „Wahrnehmungsbedingungen [...], die nach den vom Code aufgestellten Modalitäten in grafische Zeichen transkribiert worden sind“ (Eco 2002, S. 246). Im Folgenden werden einzelne Figuren (zunächst isoliert, ohne Verbindung mit den anderen) charakterisiert:

1. Der Streifen hat nicht nur eine konstante Lokalisierung auf dem Bildschirm, sondern auch eine gleich bleibende grafische Gestaltung, wie die Höhe und meistens die Farbe ${ }^{9}$.

2. Der sprachliche Text besteht aus vollständigen Sätzen, die in linearer Form emittiert werden. Wie viele „,neue Textwelten“(vgl. Eckkrammer 2002, S. 31) entzieht sich auch dieser Text den Textualitätskriterien von de Beaugrande und Dressler (vgl. de Beaugrande/Dressler 1981), indem er als nicht kohäsiv und nicht kohärent gelten muss. Erstens gibt es keine grammatischen Verknüpfungen zwischen den Sätzen. ${ }^{10}$ Zweitens bezieht sich der Inhalt der Sätze auf unterschiedlichste Themenbereiche, wodurch es keine thematischen Zusammenhänge gibt.

3. Die Geschwindigkeit ist - trotz ihrer ,Ungreifbarkeit " - ebenso als eine Figur einzustufen. Selbst Charles S. Peirce spricht vom Qualizeichen, als einer „Qualität,

\footnotetext{
${ }^{8}$ Neben Figuren und Zeichen unterscheidet Prieto auch Seme (oder ikonische Aussagen). Ein Sem definiert er als „ein besonderes Zeichen, dessen Signifikat nicht einem Zeichen entspricht, sondern einer Aussage der Sprache“ (Eco 2002, S. 236). Als ein Beispiel führt Eco ein Verkehrsschild an, das „die Fahrtrichtung untersagt“. Auch wenn es eine eindeutige Bedeutung hat, ,kann (es) nicht in Beziehung gesetzt werden zu einem äquivalenten verbalen Zeichen, sondern zu einer äquivalenten Aussage ('Fahrtrichtung untersagt" oder, Verboten, auf dieser Straße in dieser Richtung zu fahren ")“ (Eco 2002, S. 236). Diese Auffassung impliziert, dass ein Text als Ganzes keinen Zeichenwert besitzt, was jedoch als umstritten gilt (vgl. Gansel/Jürgens 2007, S. 18-22).

9 In polnischen Nachrichtensendungen mit Tickern wird die Ticker-Farbe bei besonders wichtigen Ereignissen in der Regel von der blauen in die rote umgeschaltet (vgl. Trojanowska/Francuz 2007, S. 68).

${ }^{10}$ Zusätzlich kann die Isolierung der Sätze durch ikonische Figuren, die die Sätze voneinander trennen, wie zum Beispiel Miniaturen vom Logo des Fernsehsenders, veranschaulicht werden.
} 
die ein Zeichen ist. Es kann nicht wirklich als Zeichen fungieren, ehe es nicht verkörpert ist, doch die Verkörperung hat mit seinem Zeichencharakter nichts zu tun“ (Peirce 1998, S. 123). Eco zufolge ist es „,ein bedeutungstragendes Merkmal, wie der Ton der Stimme beim Aussprechen eines Wortes, Farbe und Stoff eines Kleidungsstücks usw.“ (Eco 1977, S. 58). Auch wenn es schwer ist, der Geschwindigkeit eine selbständige Bedeutung zuzuschreiben, erscheint es als berechtigt, sie (durch Analogie) für eine ,Qualifigur' zu halten, die nach ihrer ,Transkription “ in das grafische Zeichen (den TV-Ticker) zur wichtigen ,Wahrnehmungsbedingung ‘ dieses Zeichens wird.

Ob der TV-Ticker als ein Zeichen (und nicht ein Signal) aufzufassen ist, hängt demnach in erster Linie davon ab, ob ein vereinbarter Kode vorliegt. Dabei ist nicht der sprachliche Kode gemeint, der zweifelsohne den sprachlichen Text regelt, sondern der Kode der Anwesenheit beziehungsweise Abwesenheit des Tickers. Eine derartige binäre Relation exemplifiziert Eco mit einer Signalleuchte, die das Erreichen eines bestimmten Wasserniveaus signalisieren soll:

Das Lämpchen bildet schon den Anfang eines Code:/brennendes Lämpchen/ bedeutet „Niveau $\mathrm{O}^{11}$ erreicht“", während/ausgeschaltetes Lämpchen/,Niveau unter O“ bedeutet (Eco 2002, S. 63).

Wenn der Empfänger dieses Signals ein Mensch ist, der instruiert wurde, was das brennende beziehungsweise ausgeschaltete Lämpchen bedeutet, ist es für ihn mit der Bedeutung verbunden (vgl. Eco 2002, S. 65). ${ }^{12}$ Die Vereinbarung muss jedoch nicht unbedingt durch nachdrückliches Instruieren des Empfängers erfolgen. Möglich ist auch eine ,Vereinbarung' mittels der Konvention. Das ist der Fall bei TV-Tickern, wobei es niemals mit den Empfängern geklärt wurde, dass sie sich durch ihre Anwesenheit laufend informiert fühlen sollen. Nur aufgrund eigener Erfahrung (indem sie gelernt haben, dass mit dem Ticker die gekürzten Informationen ausgedrückt sind) wird der Kode erworben.

Im Unterschied zum Signallämpchen besteht der binäre Code nicht in der Dichotomie $(+) /(-)$, sondern (+) steht für Informiertsein bei Anwesenheit des TVTickers/(0) für keine Botschaft bei seiner Abwesenheit. Es darf nämlich nicht angenommen werden, dass der Empfänger die Abwesenheit des Tickers in einer Nachrichtensendung als Nicht-Informiertsein versteht. Diese Relation lässt sich mit der Kodierung bei einem Verkehrszeichen ,LANGSAM SPIELENDE KINDER '13 vergleichen. Die Anwesenheit des Zeichens bedeutet, dass Kinder möglicherweise (auch wenn nicht unbedingt) in der Nähe der Straße spielen. Dagegen bedeutet seine Abwesenheit nicht zwangsläufig, dass es keine Kinder in der Nähe der Straße gibt.

\footnotetext{
11 In der Publikation werden die Bezeichnungen ,Niveau O` und ,Niveau 0“ eher inkonsequent gebraucht (vgl. z. B. Eco 2002, S. 49).

12 Im Falle eines maschinellen Empfängers wird die Information zwar empfangen, aber nicht mit Bedeutung gefüllt. Solche maschinenbezogenen ,Kommunikations'-Schemata gehören zum Untersuchungsgegenstand der Kybernetik (vgl. Eco 2002, S. 65).

13 Dieses Beispiel wird von de Beaugrande und Dressler zur Besprechung der Kohärenz als eines der Textualitätskriterien angeführt (de Beaugrande/Dressler 1981, S. 9).
} 
Im weiteren Teil der Zeichen-Definition von Eco wird zwischen dem Kommunikationsprozess und dem Designationsprozess unterschieden. Während jedes Zeichen den Designationswert besitzt, wird der Kommunikationswert nur den Zeichen zugeschrieben, die vom Sender bewusst kodiert sind. Um die Frage beantworten zu können, ob bei den TV-Tickern nur der Designationsprozess oder auch der Kommunikationsprozess zustande kommt, muss der Sender dieser Zeichen näher betrachtet werden. Damit der Sender bewusst kodieren kann, muss er mindestens zwei Bedingungen erfüllen: Er muss menschlich sein und den Kode kennen.

Selbst die Frage nach einem menschlichen Sender kann Zweifel verursachen. Offensichtlich ist dieser Fall nicht analog zu dem, in dem das Lämpchen signalisiert, dass das Wasserniveau 0 erreicht wurde. Dabei beschränkt sich die Rolle des Menschen darauf, die Maschine so zu programmieren, dass sie in einer bestimmten Situation mit dem Aufleuchten des Lämpchens reagiert. Die Designation kann als maschinell gelten (vgl. Eco 2002, S. 47), weil die Maschine selbst (aufgrund der Programmierung), entscheidet', wann das Lämpchen aufzuleuchten hat. Im Falle der TV-Ticker ist der Mensch kontinuierlich anwesend, weil er den Text eingibt, der im Ticker angezeigt wird. Von der ständigen Anwesenheit des menschlichen Senders zeugt auch die Tatsache, dass die Einträge im Laufe der Sendung unauffällig durch neuere ersetzt werden können (vgl. Cieszkowski 2009, S. 7).

Gleichzeitig kann man bei den TV-Tickern eher nicht von einem menschlichen Sender sprechen, der genauso wie beispielsweise bei der Chat-Kommunikation die Botschaft mithilfe der Computertechnologie überträgt. Im Chat konstituiert man die Zeichen selbst, indem man aus dem vorhandenen Sortiment von Figuren (Tastatur-Zeichen) beziehungsweise Zeichen (z. B. ikonischen Emoticons) wählt. Auch beim TV-Ticker gestaltet man die anzuzeigenden Einträge, aber sie sind keinesfalls mit einem Zeichen gleichzusetzen. Vielmehr sind sie eine zusammengesetzte Figur (die aus mehreren Tastatur-Figuren besteht), die zusammen mit dem Streifen und der Geschwindigkeit ein Zeichen bilden. Der Kode, aufgrund dessen der TV-Ticker die Bedeutung ,Informiertsein“ (+)/,Informiertsein“ (0) hat, bezieht sich nämlich auf das gesamte Zeichen und nicht auf nur eine Figur. Ohne den Streifen und die Geschwindigkeit könnten die Einträge möglicherweise eine andere Bedeutung erlangen, wie die semantische Bedeutung aufgrund des sprachlichen Kodes.

Jedenfalls darf festgestellt werden, dass der menschliche Sender das Zeichen mitgestaltet. Er gibt nämlich die Einträge ein, auch wenn ihr semantischer Inhalt von geringer Bedeutung ist. Der Streifen mit all seinen Parametern sowie die Geschwindigkeit sind dagegen voreingestellt und werden meistens maschinell generiert. ${ }^{14}$

Die zweite Frage, die in Bezug auf den eventuellen Kommunikationswert gestellt werden soll, ist, ob der menschliche Sender bewusst handelt, d. h. ob er den Kode kennt und sich der Kodierung bewusst ist. Bei der Mitgestaltung des Zeichens verwendet der Sender mindestens zwei Kodes. Auf der Textebene muss er den sprachlichen Kode aktivieren. Zwar sind die Einträge nicht zum Lesen konzipiert, aber trotzdem sind sie keine Sequenzen zufälliger Tastatur-Figuren, sondern

14 Nur in besonderen Situationen wird vom menschlichen Sender in diese Einstellungen eingegriffen (vgl. Anm. 8).

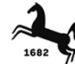


Ausformulierungen, die aufgrund des Kodes einer bestimmten Sprache verfasst werden.

Während dieser Kode im TV-Ticker , ausgeschaltet` wird $^{15}$, ist für das Zustandekommen des Kommunikationsprozesses der zweite Kode auschlaggebend. Der Sender soll nämlich wissen, dass er sich an der Designation des ,Informiertseins“ beteiligt, auch wenn das nur einen geringen Einfluss auf seine Tätigkeit hat. Sonst wäre eine solche ,kommunikative' Situation in dieser Hinsicht mit dem von Eco genannten Fall der Psychoanalyse vergleichbar. Dabei ist es möglich, dass der Patient glaubt, die Botschaft bewusst zu kodieren, während sie für den Analytiker eine Bedeutung hat, derer er sich nicht bewusst ist (vgl. Eco 1977, S. 47). Man darf jedoch annehmen, dass der Sender beim TV-Ticker auch den zweiten Kode kennt. Auch wenn er nicht nachdrücklich instruiert wurde, dass die Anwesenheit des TV-Tickers ,Informiertsein' designiert, hat er es zweifelsohne selbst durch Sozialisierung (vor allem durch eigene Erfahrungen als Empfänger der TV-Ticker) gelernt.

Dabei ist in dem Kode der Simulationsaspekt nicht enthalten. Dem Sender muss nicht unbedingt bewusst sein, dass das designierte ,Informiertsein ' simulativ ist. Eco zufolge sind die Werte wahr und falsch kein Forschungsgegenstand der Semiotik, die sich nur mit den „Bedingungen der Mitteilbarkeit und Verstehbarkeit“ befassen soll (Eco 2002, S. 72). Er illustriert diese Tatsache mit zwei Beispielsätzen: ,Napoleon starb am 5. Mai 1821 auf St. Helena' und ,Odysseus eroberte seine Herrschaft zurück, indem er alle Freier tötete'. Aus der semiotischen Sicht ist es ohne Bedeutung, dass der erste Satz historisch wahr und der zweite falsch ist. Es zählt nur

a) daß in unserer Kultur Codes bestehen, derentwegen der erste Satz verstanden wird, in der Schule gelernt wird und ,historische Wahrhaftigkeit“ konnotiert, und b) daß in der klassischen griechischen Gesellschaft Codes bestanden, durch die der zweite Satz verstanden und in der Schule gelernt wurde und ,historische Wahrhaftigkeit" konnotierte (Eco 2002, S. 72-73).

Das Zeichen ,Informiertsein“ ist demnach eine Korrelation eines Signifikanten (TVTicker) mit dem Signifikat (,Informiertsein'), das als eine kodierte kulturelle Einheit nicht unbedingt mit dem wahren Informiertsein identisch ist. Dabei ist diese kulturelle Einheit mit anderen kulturellen Einheiten, wie ,Einhorn“ oder ,Hund ‘ vergleichbar. Eco plädiert nämlich dafür, die Verifizierung eines Signifikats niemals an den Gegenstand zu binden (vgl. Eco 2002, S. 71), infolgedessen ,das Zeichen immer semiotisch autonom gegenüber den Gegenständen [ist], auf die es [eventuell] bezogen werden kann“ (Eco 1977, S. 167). Semiotisch gesehen ist es daher ohne Bedeutung, dass das ,Informiertsein “ nur simulativ ist, sowie, ob diese Tatsache dem Sender bewusst ist. Vorausgesetzt, dass er den Kode ,Informiertsein“ (+)/(0) kennt,

\footnotetext{
15 In diesem Zusammenhang spricht man u. a. von den ,virtuellen Text-Bildern“ (vgl. Cieszkowski 2009, S. 10), der ,elektronischen Bild-Schrift“ (vgl. Gwóźdź, http://kops.ub.uni-konstanz.de/static/paech/zdm/ beitrg/Gwozdz.htm) sowie von der ,,Verbildlichung der Schrift“ (vgl. Sandbothe 1996; 1997) oder auch „Ikonisierung der Textoberfläche“ (vgl. Opiłowski 2010, S. 165). Die Unterschiede in der Verwendung von Begriffen Schrift und Text in den obigen Bezeichnungen resultieren aus der Tatsache, dass man in der Sprachwissenschaft vom Text spricht, der meistens sowohl schriftlich als auch mündlich sein kann, während beispielsweise in der Philosophie zwischen Schrift (schriftlich) und Sprache (mündlich) differenziert wird (vgl. Sandbothe 1996).
} 
was mit großer Wahrscheinlichkeit der Fall ist, kann er als ein bewusster Sender gelten.

Zweifelsohne bringt die Medienentwicklung neue Herausforderungen an die Lehre von den Zeichen mit sich. Sie verlangt eine vorurteilsfreie Einstellung und eine Zeichenauffassung, die breit genug ist, um neue zeichenhafte Einheiten einzuschließen. Die durchgeführte zeichentheoretische Analyse hat nicht nur nachgewiesen, dass die TV-Ticker Zeichenwert, und zwar Kommunikationswert, haben. Sie hat auch gezeigt, inwieweit die Medien die Zeichenhaftigkeit beeinflussen können. Bisher wäre es kaum möglich gewesen, einer nach sprachlichen Regeln geformten Buchstabenabfolge die Textualität (und Zeichenhaftigkeit im Allgemeinen) abzusprechen. Im medialen Kontext kann sie dagegen - wie im TV-Ticker - zu einer für sich genommen bedeutungslosen Figur werden.

\section{Literaturverzeichnis}

Baudrillard, Jean: Agonie des Realen. Berlin 1978.

Baudrillard, Jean: Simulacra and simulation. Michigan 2006.

Brinker, Klaus: Linguistische Textanalyse. Berlin 2010.

Burger, Harald: Mediensprache. Eine Einführung in Sprache und Kommunikationsformen der Massenmedien. Berlin/New York 2005.

Champlin, Dell P./Knoedler, Janet T.: „Operating in the Public Interest or in Pursuit of Private Profits? News in the Age of Media Consolidation“. In: Journal of Economic Issues 36 (2002), S. 459-468.

Cieszkowski, Marek: Zeichen und Zeichenrelationen. Beispielhaft dargestellt an Tier-Textemen aus Friedrich Nietsches Werk „Als sprach Zarathustra“. Eine kritische Bewertung semantischer Theorien. Bydgoszcz 2001.

Cieszkowski, Marek: O zasadzie równoczesności w multiprzekazie. Manuskript 2009.

de Beaugrande, Robert A./Dressler, Wolfgang U.: Einführung in die Textlinguistik. Tübingen 1981.

Eckkrammer, Eva M.: „Brauchen wir einen neuen Textbegriff?“. In: Ulla Fix/Kirsten Adamzik/Gerd Antos/Michael Klemm (Hg.): Brauchen wir einen neuen Textbegriff? Frankfurt a. M. 2002, S. 31-58.

Eco, Umberto: Zeichen. Einführung in einen Begriff und seine Geschichte. Frankfurt a. M. 1977.

Eco, Umberto: Einführung in die Semiotik. Paderborn 2002.

Gansel, Christina/Jürgens, Frank: Textlinguistik und Textgrammatik. Göttingen 2007.

Gwóźdź, Andrzej: Die Spektakel der Schrift im Monitorstadium des Films. In: http://www.uni-konstanz.de/paech2002/zdm/beitrg/Gwozdz.htm (22.09.2013).

Harris, Roy: Signs of Writing. Routledge 1995.

Kapuścińska, Anna: „Gdy tekst staje się obrazem. Funkcja wizualna tekstu na przykładzie wybranych tekstów medialnych“. In: Prace językoznawcze 14 (2012), S. 111-118.

Marchal, Peter: „Zukünftige Programmentwicklungen des Hörfunks“. In: Joachim-Felix Leonhardt u. a. (Hg.): Medienwissenschaft. 3. Teilband. Berlin 2002, S. 2117-2126.

Opiłowski, Roman: „Wie viel Bildlichkeit steckt in der Schriftlichkeit? Zur formalen und funktionalen Kreativität in der Werbetypographie“. In: Grażyna Łopuszańska (Hg.): Mehrsprachigkeit und Mehrkulturalität der Firmenkommunikation. Fremdwörter im Bankwesendiskurs. Gdańsk 2010, S. 165174.

Otto, Kim/Scheurer, Hans/Vogel, Jochen: Das Medienlexikon. Begriffe - Zusammenhänge - Institutionen. Bonn 2012.

Peirce, Charles S.: Phänomen und Logik der Zeichen. Frankfurt a. M. 1998.

Postman, Neil: Wir amüsieren uns zu Tode. Frankfurt a. M. 2008.

Sandbothe, Mike: Bild, Sprache und Schrift im Zeitalter des Internet. [1996]. In: http://www.sandbothe.net/ 39.html (22.09.2013).

Sandbothe, Mike: Digitale Verflechtungen. Eine medienphilosophische Analyse von Bild, Sprache und Schrift im Internet [1997]. In: http://www.sandbothe.net/37.html (22.09.2013).

Schwarzkopf, Dietrich: „Organisationsstrukturen des Fernsehens“. In: Joachim-Felix Leonhardt u. a. (Hg.): Medienwissenschaft. 3. Teilband. Berlin 2002, S. 2256-2269.

„Staatsvertrag für Rundfunk und Telemedien (Rundfunkstaatsvertrag - RStV -) vom 31. August 1991, in der Fassung des Fünfzehnten Staatsvertrages zur Änderung rundfunkrechtlicher Staatsverträge (Fünf- 
zehnter Rundfunkänderungsstaatsvertrag) vom 15. bis 21. Dezember 2010“. In: Media Perspektiven. Dokumentation I (2012), S. 3-38.

Trabant, Jürgen: Elemente der Semiotik. Tübingen 1996.

Trojanowska, Agnieszka/Francuz, Piotr: „Rozumienie przekazu audiowizualnego zawierającego ,tekst taśmowy“ (TV-ticker) przez osoby zależne i niezależne od pola“. In: Piotr Francuz (Hg.): Psychologiczne aspekty komunikacji audiowizualnej. Lublin 2007, S. 67-86.

Virilio, Paul: Information und Apokalypse. Die Strategie der Täuschung. München/Wien 2000. 